\title{
THE CREATION OF SPACE VECTOR MODELS OF BUILDINGS FROM RPAS PHOTOGRAMMETRY DATA
}

Ondrej TRHAN ${ }^{*}$

\section{Abstract}

The results of Remote Piloted Aircraft System (RPAS) photogrammetry are digital surface models and orthophotos. The main problem of the digital surface models obtained is that buildings are not perpendicular and the shape of roofs is deformed. The task of this paper is to obtain a more accurate digital surface model using building reconstructions. The paper discusses the problem of obtaining and approximating building footprints, reconstructing the final spatial vector digital building model, and modifying the buildings on the digital surface model.

\section{Address}

1 Department of Surveying, Faculty of Civil Engineering, Slovak University of Technology, Bratislava, Slovakia

* Corresponding author: ondrej.trhan@stuba.sk

\section{Key words}

- RPAS photogrammetry,

- DSM,

- Building reconstruction,

- Space model.

\section{INTRODUCTION}

There have been significant developments in the field of building detection and reconstruction in the past 20 years. Various approaches with different detection and reconstruction methods have been used. However, the authors either focus on the whole building reconstruction process or only on certain parts of this process.

(Braun et al., 1995) modelled buildings on the basis of parametric volume primitives. According to (Haala and Brenner, 1997), the interpretation process in 3D building reconstruction can be simplified if digital surface models (DSM) are used in addition to or instead of image data. (Cohen and Vinson, 2002) used the method of perpendicular building detection. They used airborne images for the creation of orthophotos and DSM.

The main part of spatial city models are buildings. These buildings can be created using various methods. Digital city models consist of space building models and are mostly used by rescue systems, engineering networks, GIS or other databases (Chen et al., 2004; Nizar et al., 2006). Most of the presented works use modelling and detection data of buildings obtained by LiDAR systems (Sun, 2013; Arefi, 2009; Zhang, 2012; Rottensteiner, 2008; Demir, 2013) or by airborne photogrammetry (Scholze, 2002). In our case, we used data acquired only from RPAS photogrammetry. This photogrammetric method is widespread nowadays. This is mostly because of the need for speed and a high degree of precision along with financial demands.

RPAS photogrammetry data are used less often for this purpose (Karantzalos et al., 2015; Nex and Remondino, 2012) and have only been in use for a few years.

The main problem of modelling buildings from photogrammetric data is that the objects (buildings) are not perpendicular as they should be. Therefore, it is necessary to detect the buildings and reconstruct them in the correct way.

We used data obtained only from one system in our work, i.e., RPAS photogrammetry. We used a digital surface model in the form of pointclouds and orthophotos as the input data. This kind of solution with the approach presented is unique.

Previous articles (Trhan et al., 2016a; Trhan et al., 2016b) have already presented detection methods for building footprints, and some digital masks obtained by these methods are used in this paper in the process of approximating building borders.

\section{MATERIAL AND METHODS}

Data acquired by the Gatewing X100 RPAS system and Ricoh GR III digital camera were used for the detection and modelling of the buildings. The testing data were acquired in April 2011. The height of 
the flight was $300 \mathrm{~m}$ above ground level, and the data were supplied by an outside company. This data was used because of the different types of buildings, visible trees, and other objects near the buildings. The positional accuracy of the system was $0.11 \mathrm{~m}$, and the accuracy of the height was $0.16 \mathrm{~m}$ (Trhan, 2013). The orthophotos and DSM were created using Agisoft Photoscan Professional software with $10 \mathrm{~cm}$ GSD.

Different methods were used for the detection and reconstruction process and are described in the following chapters.

\subsection{Footprint Detection Using Watershed Segmentation}

The concept of watershed segmentation is based on visualizing an image in three dimensions. The position of a point represents pixel coordinates, and the height represents the value of the intensity in a black and white spectrum. We consider three types of points in this kind of topographic interpretation (Gonzales et al. 2009):

- points belonging to a regional minimum,

- points on which a drop of water would fall with certainty to a single minimum,

- points on which water would be equally likely to fall on more than one such minimum.

The set of points satisfying the second condition is called a catchment basin or watershed. The points satisfying the third condition form crest lines on a topographic surface and are called divide lines or watershed lines (Fig. 1)

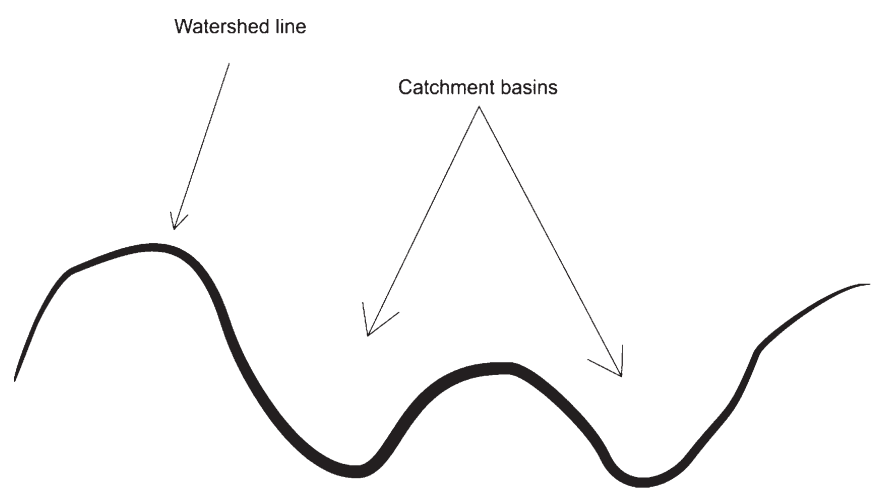

Fig. 1 Watershed segmentation situation

The principal objectives of the segmentation algorithm based on these concepts are to find the watershed lines. Assume that a hole is punched in each regional minimum and that the entire topography is flooded from below by letting water rise through the holes at a uniform rate. When the rising water is distinct, the catchment basins are about to merge, so a dam is built to prevent the merger. The flooding will eventually reach a stage when only the tops of the dams are visible above the water level. These dam boundaries correspond to the divide lines of the watersheds and are boundaries extracted by a watershed segmentation algorithm (Gonzales et al., 2009). In the watershed's segmented image this boundary is one pixel wide.

One of the key applications of watershed segmentation is in the extraction of almost uniform objects from the background. Regions characterized by small variations in intensity have small gradient values. Thus, in practise, we often see watershed segmentation applied to the gradient of an image, rather than to the image itself. Under this formulation, the regional minima of catchment basins correlate nicely with the small value of the gradient corresponding to the object of interest (Gonzales et al., 2009; Vincent and Soille, 1991).

\subsubsection{Dam construction}

Dam construction (Fig. 2) in a watershed segmentation is based on binary images, which are members of a $2 \mathrm{D}$ integer space. The simplest way to construct dams separating sets of binary points is to use morphological dilation. Let $M_{1}$ and $M_{2}$ denote the sets of the coordinates of the points in two regional minima. Then let the set of the coordinates of the points in the catchment basin associated with these two minima at stage $n-1$ of the flooding be denoted by $C_{n-1}\left(M_{1}\right)$ and $C_{n-1}\left(M_{2}\right)$. Let $C[n-1]$ denote the union of these two sets. Through this union there will be one connected component. The fact that the two connected components have become a single component indicates that the water between the two catchment basins has merged at flooding step $n$. Let this connected component be denoted $q$. Two components from step $n-1$ can be extracted from $q$ by performing the AND operation $q \cap C[n-1]$. Assume that each of the connected components is dilated by the structuring element, subject to two conditions: the dilation has to be constrained at $q$, and the dilation cannot be performed on points that would cause the sets being dilated to merge (become a single connected component) (Gonzales et al., 2009).

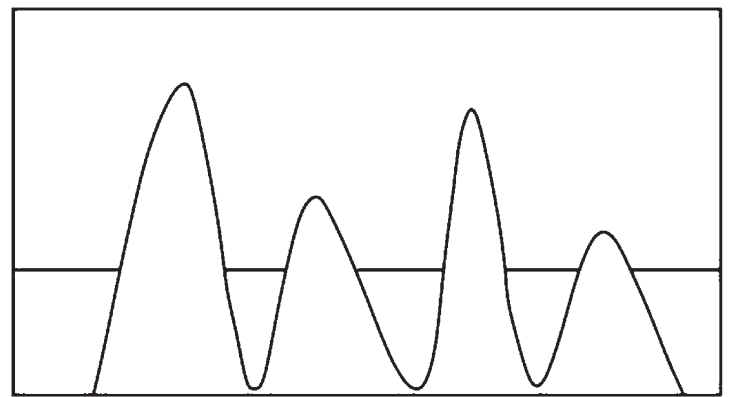

(a)

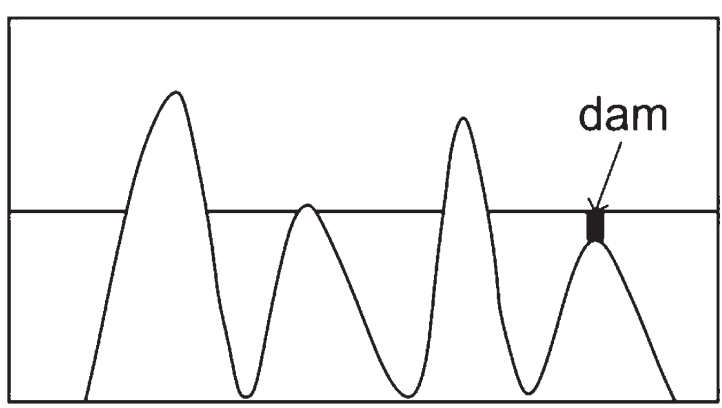

(b)

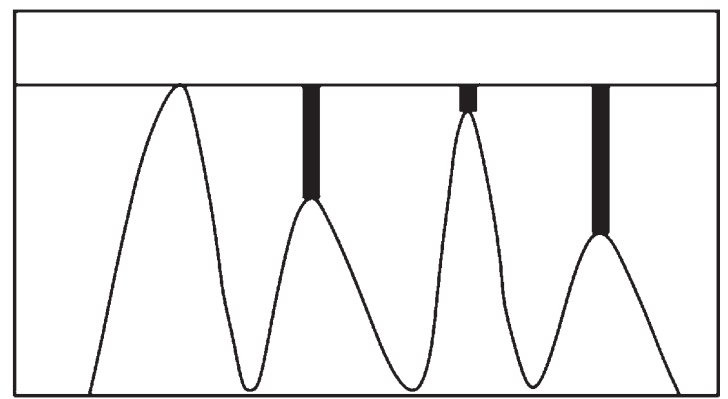

(c)

Fig. 2 Creation of a dam 


\subsubsection{Watershed segmentation algorithm}

Let $M_{1}, M_{2}, \ldots, M_{R}$ be sets denoting the coordinates of the points in the regional minima of an image $g(x, y)$. This typically will be a gradient image. Let $C(M)$ be a set denoting the coordinates of the points in the catchment basin associated with the regional minimum $M_{i}$. The notations min and max will be used to denote the minimum and maximum values of $g(x, y)$. Finally, let $T[n]$ represent the set of coordinates $(s, t)$ for which $g(s, t)<n$. That is, (Gonzales et al., 2009),

$$
T[n]=\{(s, t)) g(s, t)<n\} .
$$

Geometrically, $T[n]$ is the set of points in $g(x, y)$ lying below the plane $g(x, y)=n$. The topography will be flooded in integer flood increments, from $n=\min +1$ to $n=\max +1$. At any step $n$ of the flooding process, the algorithm needs to know the number of points below the flood depths (Gonzales et al., 2009).

Let $C_{n}\left(M_{i}\right)$ denote the set of the coordinates of the points in the catchment basin associated with the minimum $M_{i}$ that are flooded at stage $n . C_{n}\left(M_{i}\right)$ may be viewed as a binary image given by (Gonzales et al., 2009):

$$
C_{n}\left(M_{i}\right)=C\left(M_{i}\right) \cap T[n] .
$$

In other words, $C_{n}\left(M_{i}\right)=1$ at location $(x, y)$ if $(x, y) \in C\left(M_{i}\right) \wedge(x$, y) $\epsilon T[n]$; otherwise, $C_{n}\left(M_{i}\right)=0$. Next, let $C[n]$ denote the union of the flooded catchment basins at stage $n$ (Gonzales et al., 2009):

$$
C[n]=\bigcup_{i=1}^{R} C_{n}\left(M_{i}\right)
$$

Then $C[\max +1]$ is the union of all the catchment basins (Gonzales et al., 2009):

$$
C[\max +1]=\bigcup_{i=1}^{R} C\left(M_{i}\right)
$$

The elements in both $C_{n}\left(M_{i}\right)$ and $T[n]$ are never replaced during the execution of the algorithm, and the number of elements in these two sets either increases or remains the same as $n$ increases. Thus, it follows that $C[n-1]$ is a subset of $C[n]$, and according to Eqs. (1) and (2), $C[n-1]$ is a subset of $T[n]$. We can therefore say that each connected component of $C[n-1]$ is contained in exactly one connected component of $T[n]$ (Gonzales et al., 2009).

The algorithm for finding the watershed lines is initialized with

$$
\mathrm{C}[\min +1]=\mathrm{T}[\min +1] .
$$

The algorithm then proceeds recursively, computing $C[n]$ from $C[n-1]$. Let $Q$ denote the set of connected components in $T[n]$. Then, for each connected component $q \in Q[n]$, there are three possibilities (Gonzales et al., 2009):

- $q \bigcap C[n-1]$ is empty,

- $q \bigcap C[n-1]$ contains one connected component of $C[n-1]$,

- $q \bigcap C[n-1]$ contains more than one connected component of $C[n-1]$.

The construction of $C[n]$ from $C[n-1]$ depends on which of these three conditions holds. Condition one occurs when a new minimum is encountered. Condition two occurs when $q$ lies within the catchment basin of some regional minimum, in which case $q$ is incorporated into $C[n-1]$ to form $C[n]$. Condition three occurs when all or part of a ridge separating two or more catchment basins is encountered. Further flooding would cause the water levels in these catchments to merge. Thus a dam must be built. The efficiency of the algorithm is improved by only using the values of $n$ that correspond to the existing intensity values in $g(x, y)$ (Gonzales et al., 2009).

\subsubsection{The use of markers}

The direct application of the watershed segmentation algorithm in this form generally leads to oversegmentation due to noise and other local irregularities of the gradient. Oversegmentation can be serious enough to render the results of the algorithm virtually useless. A practical solution to this problem is to limit the number of allowable regions by incorporating a preprocessing stage designed to bring additional knowledge into the segmentation procedure.

The approach used to control oversegmentation is based on the concept of markers. A marker is a connected component belonging to an image. We know of internal markers associated with objects of interest and external markers associated with the background. The procedure for marker selection will typically consist of two principal steps: the preprocessing and definition of a set of criteria that the markers must satisfy.

The selection of the marker can range from simple procedures based on intensity values and connectivity to more complex descriptions involving size, shape, location, relative distances, texture content, etc. (Gonzales et al., 2009).

\subsection{Approximating Building Footprints}

Building footprints have to be extracted and simplified before modelling spatial buildings. The boundaries of buildings often contain minor protuberances or other irregularities that can be removed without big effects on the final shape of the building. In general, the approximation of the building boundary is a process when the entry boundary is simplified and all the morphological features of the building are maintained.

The buildings are often formed by a relatively simple ground plan which consists of straight lines and right angles. However, it is necessary to maintain several conditions. Significant parts of the building must be preserved. Very small structures or short edges can be removed. The symmetry of the buildings must be preserved. The number of the resulting linear segments should be minimized. The distance between the specified points and the edge should be as small as possible to prevent incorrect displays. The normal vector of the resulting border should be similar to the input line. The area of the original and resulting footprint should be as similar as possible. The algorithm should be fault tolerant. It should be possible to control the smoothing and degree of generalization of the model. The angles which are close to $90^{\circ}$ or $270^{\circ}$ are considered to be these angles (Arefi, 2009)

In principle, we can consider two solutions. The first solution is a simple adaptation of polygons to the resulting area. To this solution we might include an iterative construction of a minimum bounding rectangle (MBR). The main principle of MBR is the hierarchical fitting of rectangular polygons to the area selected. A second suitable method may be a Ramer algorithm (Ramer, 1972). The detection of lines, which can be detected by Hough transform or RANSAC (Arefi, 2009), is used in this method.

We decided to use the MBR method and Kovesi's method (Kovesi, 2017) to search for the lines. The MBR method was used for build- 
ings with angles close to $90^{\circ}$. Kovesi's method was used for other buildings.

\subsection{Creation of the Building Models}

Another important step in the process of the detection and reconstruction of buildings is the creation of an accurate building model. Buildings can be reconstructed in various Levels of Detail (LOD). Based on this, building models can be represented in space by varying the amount of necessary information and their details. Different LOD are defined in CityGML (Kolbe et al., 2006), which lists different levels of categorization. CityGML is an open data model and format based on a GML base for the storage and exchange of virtual spatial models of cities (OCG, 2017). We know five levels. The first level is called LOD0 and is a 2.5D representation of DSM. This stage is about displaying the orthophoto into a model. LOD1 is a rectangular building model without roof structures and texture. We get LOD2 by enriching the roofs. LOD3 is made by adding architectural information with wall details and smaller structures on the roof display. The last class is LOD4, which contains all of the building information, including the interior (Gonzales et al., 2009; Mackaness et al., 2009; Kim, 2012). The differences between the LODs are in Fig. 3.

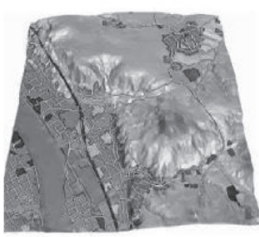

LODO

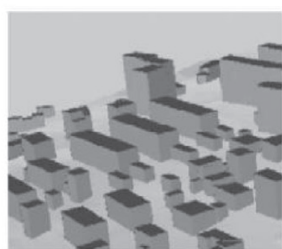

LOD1

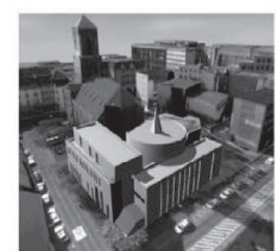

LOD2
LOD3
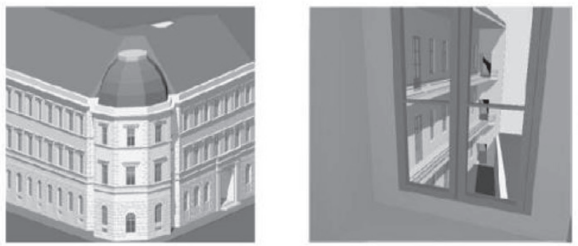

Fig. 3 The representation of LOD (Kolbe et al., 2006)

\subsubsection{Detection of the roof ridge}

It is important to find and determine roof ridges when creating spatial vector building models. First, it is necessary to determine the type of roof. In this paper, all the roofs are saddle roofs.

Several methods can be used to define roof ridges. The first method uses geodesic morphology. Locating and determining roof ridges is significant, as the resulting quality of the building model depends on the quality of this detection. Ridge lines are the basis for the division of buildings into separate parts and form the basis of modelling these parts (Arefi and Reinartz, 2011).

The method used employs searching for the regional maxima of DSM. These maxima serve as a basis for the creation of roof ridges. This process is based on an algorithm, which uses image reconstruction involving geodesic morphological dilation (Arefi and Hahn, 2005). Geodetic dilation differs from classical dilation in that it uses images and structured elements in the filtration process. In geodetic dilation the dilated image masks use a predefined masking image. In most cases, the original image is defined as a marker image with a certain offset (in our case, it is the reduction of the value height at each point), and it represents the original DSM (Fig. 4).

The input image, which is called a marker $(\mathrm{J})$, is dilated. Next, the image is limited by the masking image (I). The results of the geodetic dilation are shown in Fig. 4 (d). In the case of insufficient results (it does not show only the roof ridge), it is possible to apply iterations. The number of iterations depends on the input differences. The results obtained using these steps are called "'marker reconstruction using a geodetic dilation mask" (Arefi and Hahn, 2005).

Based on the information presented, geodetic dilation $\delta_{I}$ and the image reconstruction are defined as:

$$
\begin{gathered}
\delta_{I}^{(1)}(J)=(J \oplus B) \wedge I, \\
\delta_{I}^{(n)}(J)=\delta_{I}^{(1)}(J) \circ \delta_{I}^{(2)}(J) \circ \ldots \circ \delta_{I}^{(n)}(J) .
\end{gathered}
$$

Equation (7) defines the morphological reconstruction of the marker image $(\mathrm{J})$ based on the geodetic dilation.

The second method to find the roof ridges was to use the DSM's slope at each point. We used the formula:

$$
\text { slope }=\sqrt{\left(\frac{D E M_{i-1, j}-D E M_{i+1, j}}{2 h}\right)^{2}+\left(\frac{D E M_{i, j-1}-D E M_{i, j+1}}{2 h}\right)^{2}}
$$

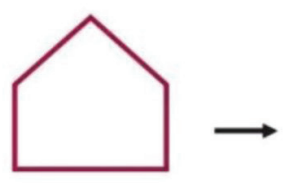

(a) Marker (J)

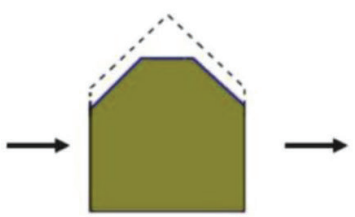

(e) Geodesic dilation of size 2

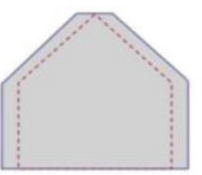

(b) Dilation of (J) (c)

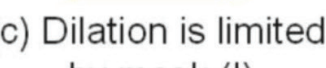

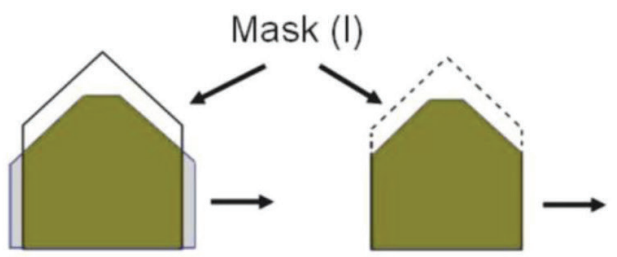

(d) Geodesic dilation of size 1

by mask (I)

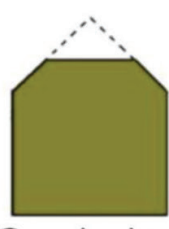

$=>$ Image reconstruction by Geodesic dilation

(f) Geodesic dilation of size 3 (g) Geodesic dilation

of size 4

Fig. 4 Process of geodetic dilation (Arefi and Hahn, 2005) 

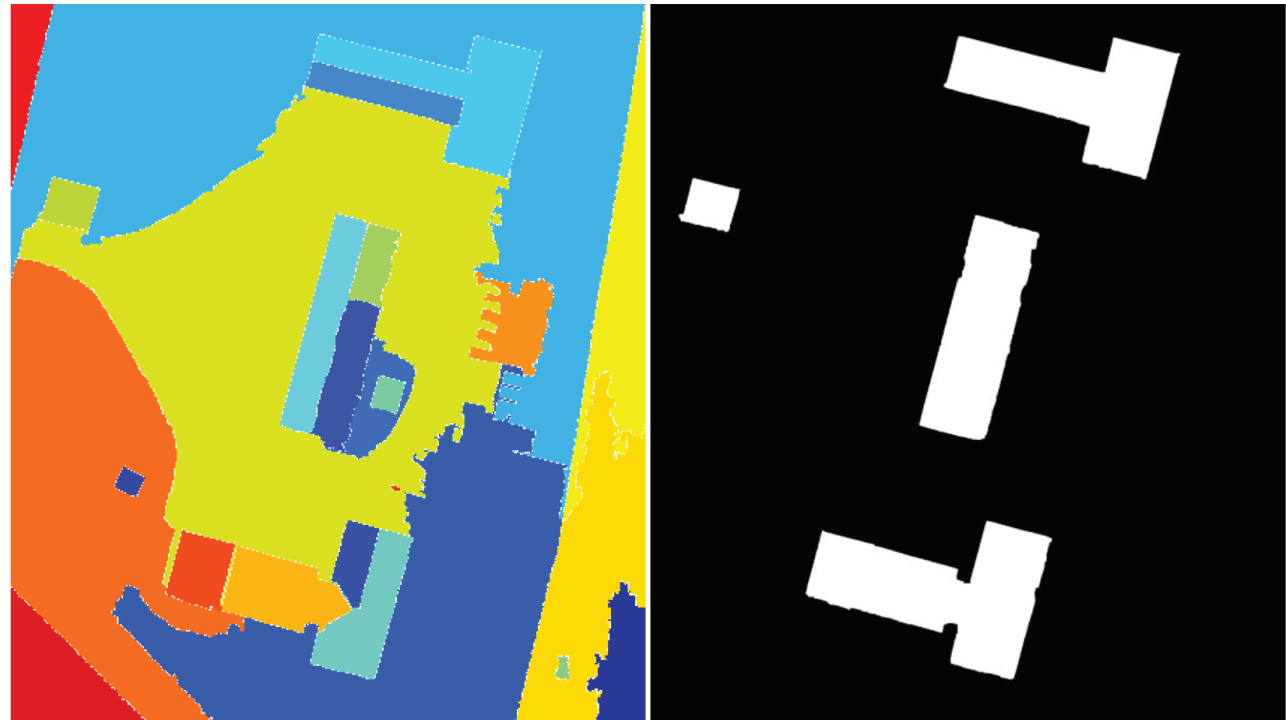

Fig. 5 The results of the watershed segmentation (left) and the digital mask obtained (right)

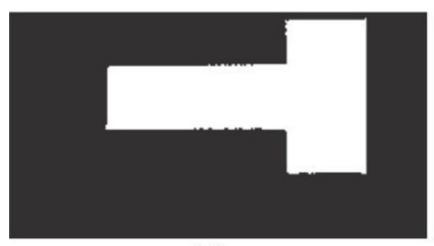

(1)

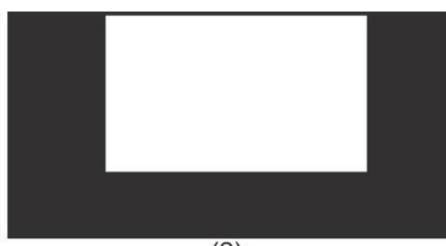

(2)

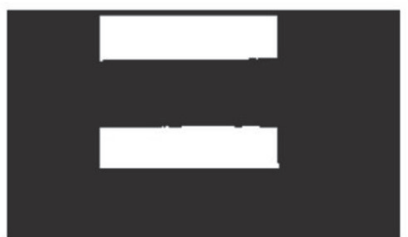

(3)

Fig. 6 Rotated building (1), minimum bounding rectangle (2), MBR without the building (3)

The second method was used to compare different methods and their results.

\section{RESULTS}

\subsection{The Use Of The Watershed Segmentation Algorithm Employing Markers}

Watershed segmentation is most suitable for images where the background and foreground locations can be identified from the image. In the first step of the algorithm the image is transformed to grayscale. In the second step, Sobel edge detection is used, and the image is filtered to compute the image gradient's magnitude. The biggest gradient is at the areas of the borders of objects.

In the next step, the foreground of the objects in the image is highlighted. To achieve this, morphological techniques called 'the opening by reconstruction" and "the closing by reconstruction" were used to clean the image. These operations create a flat maxima inside each object. These morphological operations are used because they are more effective at removing small blemishes from the objects without affecting their overall shape.

Computating the regional maxima is then performed to obtain good foreground markers. With this step, the segmented parts of the image are marked. The next step is to compute the markers from the background. These markers are thinned into lines, because they are too close to the edges of the objects we are trying to segment. This can be done by computing the watershed transformation of the distance transformation of the background marker images and then looking for the watershed ridge lines of the results. The image is subsequently modified, so it only shows regional minima in certain desired locations. The image of the gradient magnitude is also modified, and the watershed segmentation is finally computed.

Watershed segmentation results in segmented areas being shown as building borders (Fig. 5 left). These areas consist of areas with sharing features. The selection of the building is done using a digital mask, which we previously obtained (Trhan et al., 2016a; Trhan et al., 2016b). Subsequently, we obtained a digital mask with the buildings. This digital mask has to be modified from the rest of the morphological operations. We then get a final digital mask. It can be used in the following steps of approximating the building's edges (Fig. 5, right).

\subsection{Minimum Bounding Rectangle Method}

This method assumes the same orientation of the coordinate system of an image as the coordinate system of a polygon. The digital mask is divided into individual objects. In other words, each building has its own digital mask. Edge detection is applied to these digital masks. The lines are then computed using Hough line detection, the advantage of which is the computation of the azimuth of each line. The average azimuth from all the lines is computed, and the result is the building's orientation. Using this angle, the building is rotated to the coordinate system of a polygon.

The rotated building is iteratively bounded. The result is a filled rectangle, which covers the area of the building. The digital mask is subtracted from this rectangle, and the results are small parts. These parts are again bounded, and the process continues until it reached the statements requested. Finally, all the parts of the building's rectangles are connected, and we obtain a building with perpendicular and straight borders (Fig. 6). The building is rotated back to its coordinate system. Each building is computed alone, and all the results are joined together at the end. 


\subsection{Kovesi's Straight Line Detection Method}

Kovesi's algorithm (Kovesi, 2017) is applied to buildings which do not have perpendicular lines. The input parameters of the algorithm edgelink (Kovesi, 2017) are the edge image and minimal length of the segments. The outcome is a subset of cells with a list of the starting and ending points of the lines. The results can be viewed and applied to the orthophoto. The function joins the edge points to the list of coordinate pairs.

The second function used, which is used to apply lines to the orthophoto, is called drawedgelist (Kovesi, 2017). The border obtained by this function is applied to the previously acquired building borders. We obtain the completed digital mask, which can be used in the next steps (Fig. 7).

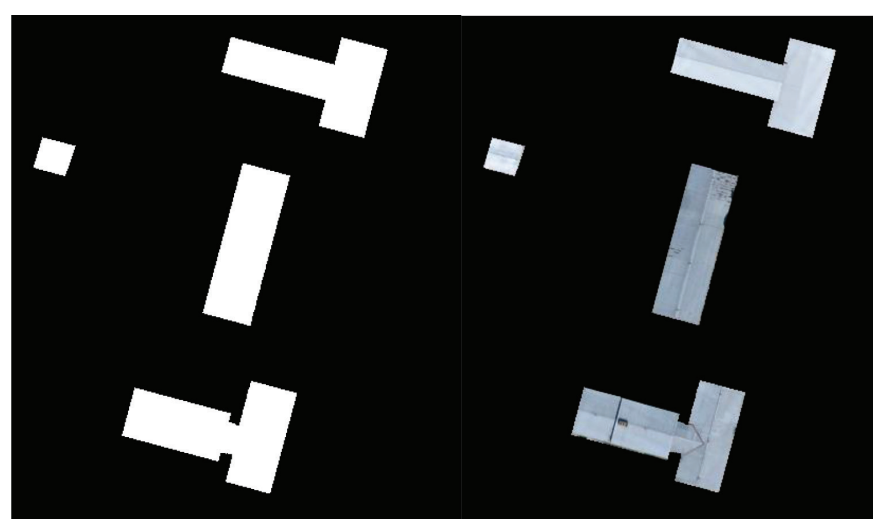

Fig. 7 The digital mask obtained by the MBR method and Kovesi 's method (left); the digital mask applied to the orthophoto (right)

\subsection{Creating the Building Models}

In this step, we modify the pointcloud with the digital mask obtained. The resulting digital mask is applied to the DSM, and only the values of the heights in the area of this mask are left. The benefit of the digital mask is that the dimensions of the orthophoto and digital model are the same. The results of this application are shown in Fig. 8(2). The heights of the buildings were calculated as the average heights of the buildings in the mask. The result of this process is a box model with an approximated flat roof. The buildings in Fig. 8(1)

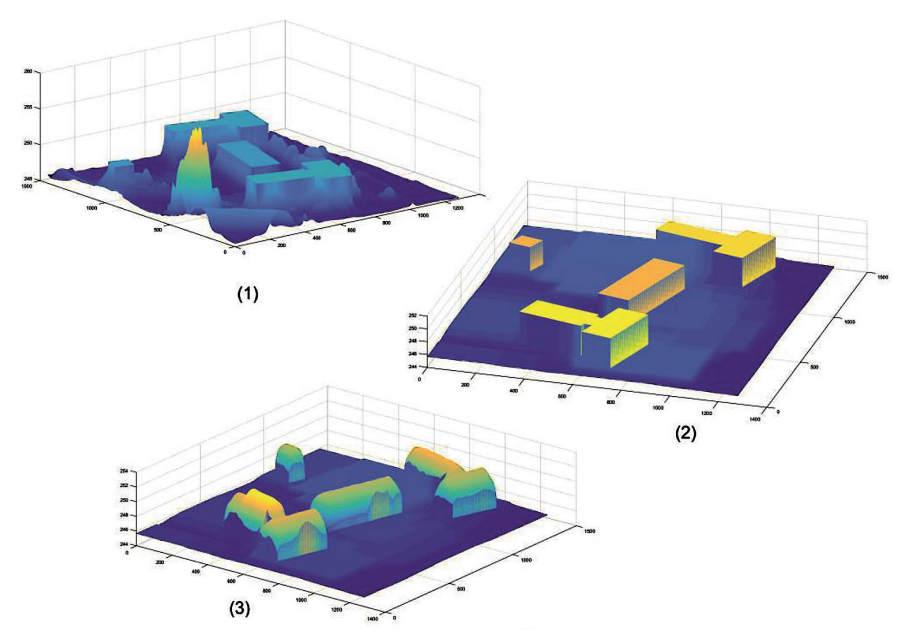

Fig. 8 LOD building models are located on DSM; the buildings in Fig. 8(2) are located on DTM; and in Fig. 8(3) the actual roof heights from the measurements in the digital mask area are preserved.

\subsection{Results of the Building Ridge Detection}

Using the knowledge gained from chapter 2.3.1, we employ an algorithm using the MATLAB software for ridge detection. The detected ridges are shown in Fig. 9, left.

Equation (8) was applied to the DSM, and the slope values were obtained. Slope $5^{\circ}$ was considered as an edge slope. After the application of this value to the data, the digital mask was obtained (Fig. 9, right). The results show that the methods used were correct.
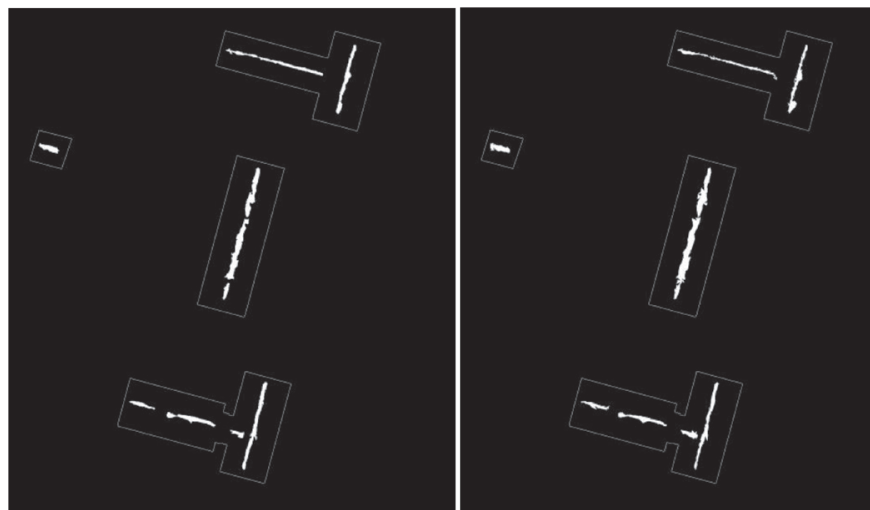

Fig. 9 The results of the ridge detection using geodetic dilation (left) and the slope (right)

\subsection{Modelling the Building}

The final step of this process was modelling the building. The modelling consists of determining the height of the building edges, the ridge area, and the placement of the building on the digital model. The average height of the building's borders was computed using DSM. The average ridge height was also computed using DSM for each part of the ridge. A new DSM was computed using the general plane equation:

$$
a x+b y+c z+d=0 \text {. }
$$

MATLAB software and the nodes and edges principle were used for modelling the buildings. Each point of the new DSM in the selected part of the roof was computed by equation (9). The results of the reconstruction process are the vector building model and the new DSM with the corrected building roofs. This model satisfies the conditions of LOD2. The resulting models are shown in Fig. 10.

\section{DISCUSSION}

In this article the process of the detection and modelling of buildings is showed. We used data obtained only by one source (RPAS photogrammetry); the DSM and orthophoto are used. The process of the work presented uses various methods for different parts of the detection and reconstruction of the buildings. These methods are mostly used on data obtained by laser scanning (Sun, 2013; Arefi, 2009; Zhang, 2012; Rottensteiner, 2008; Demir, 2013) and are usually used separately in global solutions. In our work we used a combination of these methods and applied them to the RPAS photogrammetry data. 


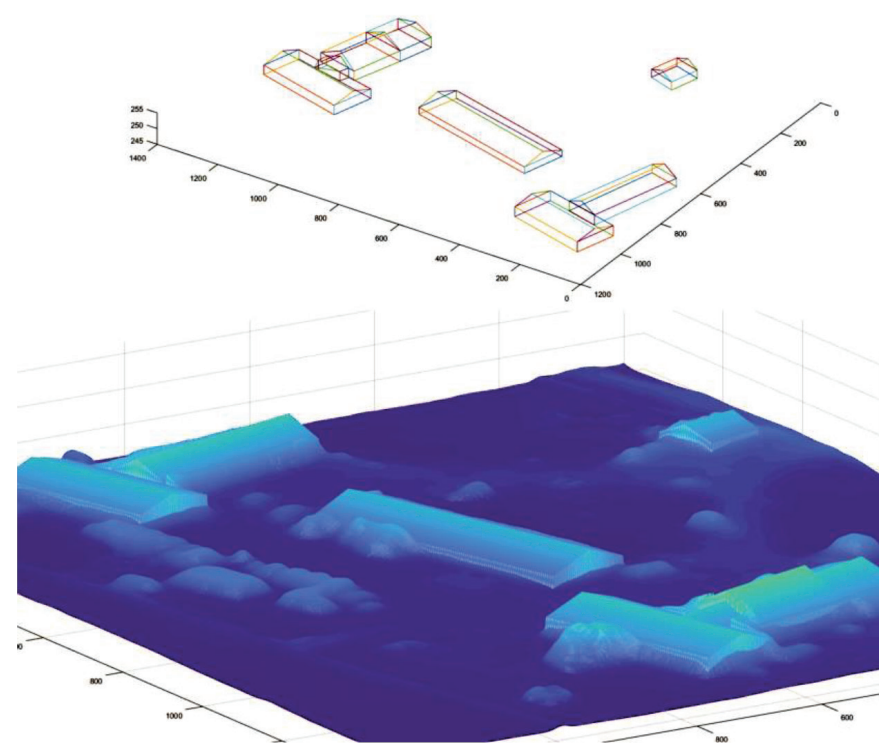

Fig. 10 The resulting vector model (above) and edited DSM (below)

The final results of the whole process are the digital vector model of the buildings and an edited DSM, where the buildings have perpendicular walls, and the roofs are straight. The problems involving the RPAS photogrammetry results of the buildings presented were removed. This edited digital model can be used for true orthophoto creation, modelling of cities, mapping applications or other GIS applications.

\section{CONCLUSION}

The aim of this article was to present the methodology for the creation and editing of the DSM obtained using RPAS photogrammetry data. In the first part of the article we focused on the acquisition of the building footprints using watershed segmentation; the edges obtained using this method are not straight and have to be adjusted. This adjustment is performed by two methods. The use of these methods depends on the angle between the building's edges. The MBR method is used for edges with angles close to $90^{\circ}$, and Kovesi's method is used for other buildings. Based on these adjusted edges, a LOD1 model was created. The roof ridges were then detected. They were the basis for the reconstruction of the building in a LOD2 specification. According to this specification, the spatial vector model using the nodes and edges methodology is created. The DSM is modified using the general plane equation into the new DSM with straight roofs. 


\section{REFERENCES}

Arefi, H. (2009) From LIDAR Point Clouds to 3D Building Models: dissertation. Munich: Universität der Bundeswehr Munchen. 127 pp.

Arefi, H. - Hahn, M. (2005) A Morphological Reconstruction Algorithm for Separating Off- Terrain Points from Terrain Points in Laser Scanning Data. International Archives of Photogrammetry, Remote Sensing and Spatial Information Sciences, Vol. 36, p. $120-125$.

Arefi, H. - Reinartz, P. (2011) Building Reconstruction from Worldview DEM Using Image Information. Tehran.

Braun, C. - Kolbe, T. H. - Lang, F. - Schickler, W. - Steinhage, V. - Cremers, A. B. et al. (1995) Models for photogrammetric building reconstruction. Computer and Graphics, 19 (1), p. 109118.

Cohen, L. D. - Vinson, S. (2002) Segmentation of complex buildings from aerial images and $3 D$ surface reconstruction. In: Proceedings of the 6th IEEE Workshop on Application of Computer Vision. IEEE, 2002. ISBN 0-7695-1858-3/02. p. 1-5.

Demir, N. (2013) Combination of airborne laser and image data for building detection and $3 \mathrm{D}$ roof reconstruction: dissertation. $\mathrm{Zu}-$ rich: ETH. 149 pp.

Gonzales, R. C. - Woods, R. E. - Eddins, S. L. (2009) Digital Image Processing Using Matlab. Gatesmark Publishing, ISBN 9780-9820-8540-0, $827 \mathrm{pp}$.

Haala, N. - Brenner, C. (1998) Interpretation of urban surface models using $2 D$ building information. Computer Vision and Image Understanding, 72 (2), pp. 204-214.

Chen, L. - Teo, T. - Rau, J. - Liu, J. - Hsu, W. (2004) Building reconstruction from LiDAR data and aerial imagery. In: Proceedings of the IEEE International Geosience and Remote Sensing Symposium. vol. 4, pp. 2846-2849.

Karantzalos, K. - Koutsourakis, P. - Kalisperakis, I. - Grammatikopoulos, L. (2015) Model-based Building Detection from Low-cost Optical Sensors Onboard Unmanned Aerial Vehicles. In: The International Archives of the Photogrammetry, Remote Sensing and Spatial Information Science. Vol. 40 - 1, International Conference on Unmanned Aerial Vehicles in Geomatics, 30 Aug - 02 Sep 2015, Toronto, Canada. 5 pp.

Kim, K. (2012) 3D Building Reconstruction from Airborne Laser Scanning Data: dissertation. West Lafayette, Indiana: Purdue University, $133 \mathrm{pp}$.

Kolbe, T. H. - Groger, G. - Plumer, L. (2006) City GML - Interoperable Acces to $3 D$ City Models. Geoinformation for Disaster
Management. In: Proceedings of the 1st International Symposium in Geo-Information for Disaster Management. Delft, The Netherlands, March 21-23, Delft.

Kovesi, P. (2017) MATLAB and Octave Functions for Computer Vision and Image Processing. Available at: http://www.peterkovesi.com/matlabfns/index.html. (accessed at 04/03/2017)

Mackaness, W. A. - Ruas, A. - Sarjakovski, L. T. (2009) Generalisation of Geographic Information: Geographic Modelling and Applications. Elsevier, $386 \mathrm{pp}$.

Nex, F. - Remondino, F. (2014) UAV for 3D mapping applications: a review. Applied Geomatics. Vol. 6, Iss. 1, 2014, p. 1-15.

OCG (2017) CityGML. Available at: http://www.opengeospatial.org/ standards/citygml. (accesed at 04/03/2017)

Ramer, U. (1972) An Iterative Procedure for the Polygonal Approximation of Plane Curves. Computer Graphics and Image Processing. No. 1, p. 244-256.

Rottensteiner, F. (2008) Automatic Extraction of Buildings from Airborne Laserscanner Data and Aerial Images: dissertation. Vienna: TU Vienna. 148 pp.

Scholze, S. (2002) A Probabilistic Approach to Building Roof Reconstruction: dissertation. Zurich: Swiss Federal Institute of Technology Zurich, $210 \mathrm{pp}$.

Sun, S. (2013) Automatic 3D Building Detection and Modeling from Airborne liDAR Point Clouds: dissertation. Rochester: Rochester Institute of Technology, $157 \mathrm{pp}$.

Trhan, O. (2013) Topographic mapping with system Gatewing X100: diploma thesis. Bratislava: SUT in Bratislava. 72 pp.

Trhan, O. - Fraštia, M. - Marčiš, M. (2016a) The creation of spatial building models using UAV photogrammetry data. Cartographic Letters, 24 (1), p. 35-45.

Trhan, O. - Fraštia, M. - Marčiš, M. (2016b) Building Models Created from UAV Photogrammetry Data. 16th International Multidisciplinary Scientific GeoConference SGEM 2016. Book 2, Vol. II, ISSN 1314-2704, p. 823-830

Vincent, L. - Soille, P. (1991) Watershed in Digital Spaces: An Efficient Algorithm Based on Immersion Situations. IEEE Transactions on Pattern Analysis and Machine Intelligence, Vol. 13. No. 6., p. 583-598.

Zhang, N. (2012) Automated Plane Detection and Extraction from Airborne Laser Scanning Data of Dense Urban Areas: diploma thesis. Lund, Sweden: Lund University, 98 pp. 